\title{
Enfermedades pulmonares difusas relacionadas al tabaco
}

\author{
ANDRÉS PINTO S.*, ESTEFANÍA PALMA R.**, EDUARDO SABBAGH P.***, \\ CRISTINA FERNÁNDEZ F.***, ÁLVARO UNDURRAGA P.*** y JUAN CARLOS RODRÍGUEZ D.***
}

\section{Smoking related interstitial lung disease}

Inhalation of tobacco smoke is a risk factor for developing respiratory diseases as chronic obstructive pulmonary disease, lung cancer and many cardiovascular diseases. Recently, a new group of interstitial lung diseases (ILD) related to cigarette smoking (SR-ILD) have been described. This group includes pulmonary Langerhans cell histiocytosis, respiratory bronchiolitis, smoking-associated interstitial fibrosis, desquamative interstitial pneumonia. The diagnosis is usually difficult, and the use of some clinical clues, high-resolution computerized tomography, and histopathologic findings in lung biopsy could help to differentiate between the various entities. We present a report of clinical cases of patients with SR-ILD seen in our center, and a review of the literature of the above entities.

Key words: Smoking; Lung disease, interstitial; Histiocytosis, Langerhans-Cell; Interstitial Pneumonitis, Desquamative,Familial; Bronchiolitis.

\section{Resumen}

La inhalación del humo de tabaco es un factor de riesgo conocido para el desarrollo de enfermedades respiratorias como la enfermedad pulmonar obstructiva crónica, el cáncer pulmonar y algunas enfermedades cardiovasculares. Se ha descrito un grupo de enfermedades pulmonares difusas (EPD), particularmente asociadas al tabaquismo (EPD-TBQ), entre ellas, la histiocitosis pulmonar de Langerhans (PLCH), la bronquiolitis respiratoria (BR), la neumonía intersticial descamativa (DIP) y recientemente la fibrosis intersticial relacionada a tabaco (SRIF). El diagnóstico suele ser complejo, y la utilización de algunas claves diagnósticas, en conjunto a la tomografía computarizada de tórax de alta resolución y los hallazgos histopatológicos de la biopsia pulmonar, pueden ayudar a diferenciar entre las distintas entidades. Se presenta a continuación, una serie de viñetas clínicas de pacientes con EPD-TBQ, atendidos en nuestro centro, y una revisión de la bibliografia sobre cada una de ellas.

Palabras clave: tabaquismo; enfermedad pulmonar intersticial; Histiocitosis, células de Langerhans; neumonía intersticial descamativa, Familiar; Bronquiolitis.

\section{Introducción}

El concepto de enfermedades pulmonares difusas, es un concepto radiológico de aplicación clínica que engloba a una serie de enfermedades caracterizadas en la radiología por opacidades bilaterales que comprometen los campos pulmonares en forma extensa. También se las denomina enfermedades intersticiales, aunque no siempre hay sólo compromiso intersticial, pues también puede existir daño alveolar, bronquiolar y vascular.

El tabaco es un problema de salud pública y una causa mayor de morbimortalidad en Chile y el mundo. Si bien su asociación con enfermedades cardiovasculares, con la enfermedad pulmonar obstructiva crónica y el cáncer es ampliamente reconocida, durante los últimos años

\footnotetext{
* Médico en formación de Medicina Interna, Hospital del Salvador, Facultad de Medicina Universidad de Chile.

** Médico en formación de Medicina Interna, Facultad de Medicina Universidad de Los Andes.

*** Instituto Nacional del Tórax.
} 
existe cada vez mayor evidencia de su asociación con otro grupo de patologías, incluyendo las enfermedades pulmonares difusas relacionadas al tabaco (EPD-TBQ). El fumar es un factor de riesgo reconocido tanto para el desarrollo como para la progresión de este grupo de enfermedades ${ }^{1-3}$.

La inflamación, destrucción, remodelamiento y reparación provocados por el consumo de tabaco, son reflejados radiológica e histológicamente como una variedad de alteraciones en la vía aérea y el parénquima pulmonar. Estas alteraciones se han descrito tradicionalmente como enfermedades separadas incluyendo enfisema, bronquiolitis respiratoria, neumonía intersticial descamativa, histiocitosis pulmonar de Langerhans, entre otras $^{4}$.

El humo de cigarrillo produce acumulación de células inflamatorias en la vía aérea pequeña, espacios alveolares e intersticio, ya sea por el aumento de mediadores proinflamatorios que reclutan a estas células, como a alteraciones en la apoptosis de ellas y aumento de su sobrevida. Además, induce la producción del factor de crecimiento transformante TGF- $\beta 1$, un conocido mediador en los procesos de fibrosis. Otros mecanismos, como el acortamiento de los telómeros, un marcador de senescencia, que se ha documentado en células epiteliales alveolares en fibrosis pulmonar idiopática, o alteraciones en la regulación de la autofagia, serían favorecidos por el tabaquismo ${ }^{5-7}$.

\section{Histiocitosis de Langerhans}

\section{Caso clínico}

Mujer de 52 años, costurera, diabética insulinorequirente usuaria de glibenclamida e insulina, hipertensa usuaria de enalapril, atenolol y nifedipino, tabaquismo suspendido hace 2 años (índice paquete año-IPA $=25$ ). Consulta por cuadro de 6 meses de evolución caracterizado por disnea mMRC 2, tos seca, baja de peso de $12 \mathrm{~kg}$, dorsalgia, sudoración nocturna y fiebre hasta $39^{\circ} \mathrm{C}$ intermitente. Recibió múltiples tratamientos antibióticos de forma ambulatoria sin resultado, por lo que se deriva a nuestro centro.

Al examen físico no presenta desaturación a $\mathrm{FiO}_{2}$ ambiental, con crépitos bilaterales en los tercios basales, sin acropaquia.

TC Tórax (Figura 1) evidenció imágenes nodulares y quísticas de predominio superior.

Pruebas de función pulmonar (PFP): Espirometría: CVF $2.790 \mathrm{~mL}(76 \%) \mathrm{VEF}_{1} 2.320 \mathrm{~mL}$ (82\%) $\mathrm{VEF}_{1} / \mathrm{CVF} 83 \%$. Volúmenes pulmonares: CPT 4,14 L (82\%), VR 1,47 L (81\%), VR/CPT
$35 \%$ (96\%). Capacidad de difusión de monóxido de carbono (DLCO): 15,02 $\mathrm{mL} / \mathrm{min} / \mathrm{mmHg}$ (60\% del teórico).

Prueba de caminata de seis minutos: $404 \mathrm{~m}$ (78\%) no presenta hipoxemia con el ejercicio $\left(\mathrm{PaO}_{2} 81-76 \mathrm{mmHg}\right)$ pero aumentó el gradiente alvéolo-arterial (A-a) $0_{2}$ de 16,9 a 29,3 mmHg.

Se realiza fibrobroncoscopía con biopsia transbronquial, la cual era compatible con histiocitosis pulmonar de Langerhans (Figura 2 A-B-C-D).

\section{Comentarios}

La histiocitosis de Langerhans ( $\mathrm{LCH})$ es un desorden raro caracterizado por infiltración y formación de nódulos constituidos por diversas células incluyendo algunas con fenotipo de células de Langerhans (CL) y otras células inflamatorias agudas y crónicas ${ }^{8}$. La LCH puede comprometer diversos órganos y sistemas, incluyendo pulmón, hueso, piel, ganglios linfáticos, sistema nervioso central, médula ósea, hígado y bazo. El compromiso pulmonar por LCH (PLCH) es más común en adultos y como compromiso aislado, pero puede presentarse como parte de una $\mathrm{LCH}$ sistémica ${ }^{9}$.

La PLCH es considerada una enfermedad rara, reportándose en un 4-5\% de las biopsias quirúrgicas de estudio de EPD. Ocurre casi únicamente en fumadores o ex fumadores ${ }^{10-12}$, principalmente adultos jóvenes, sin mayor predilección por $\operatorname{sexo}^{13}$.

Si bien un porcentaje puede presentarse de forma asintomática, lo más común es la presencia de tos seca y disnea de esfuerzo, describiéndose con cierta frecuencia además episodios de neumotórax espontáneo y dolor torácico, mientras que la fiebre y el compromiso del estado general son $\operatorname{raros}^{12-14}$.

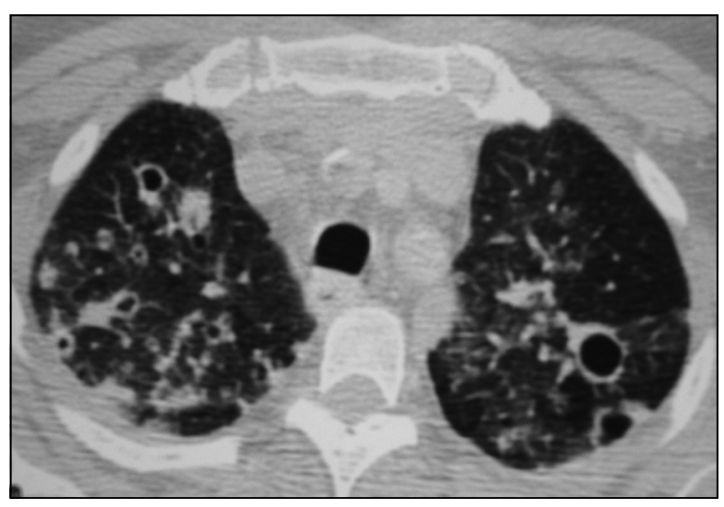

Figura 1. TC Tórax sin contraste: imágenes nodulares y quísticas de predominio superior, típicas de la PLCH (Compromiso pulmonar por histiocitosis de Langerhans). 


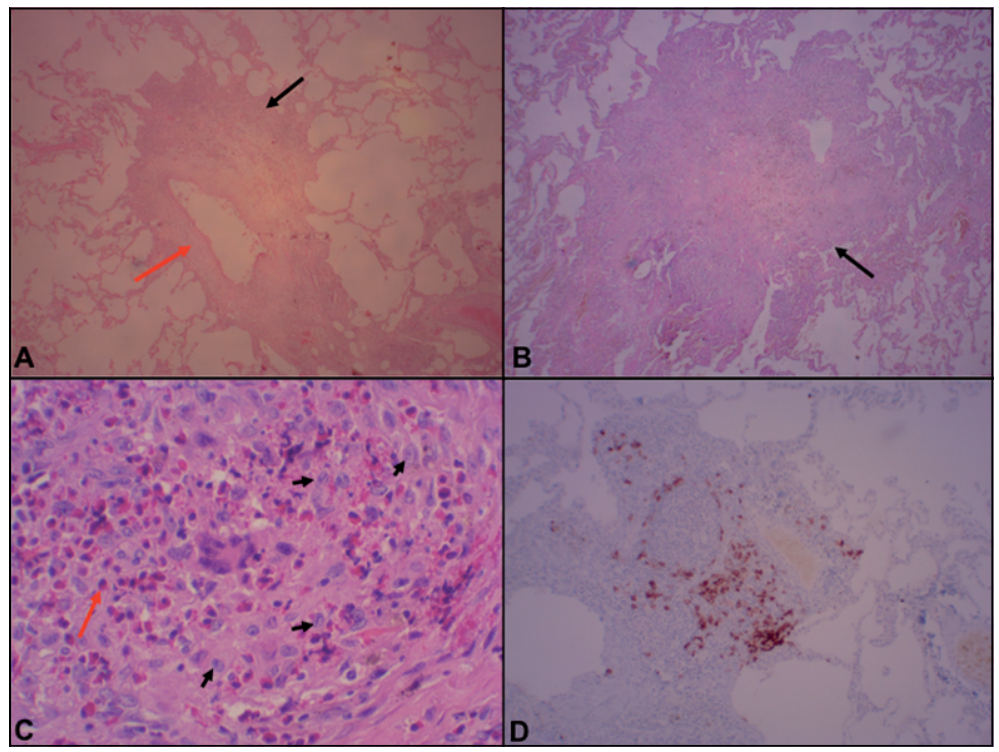

Figura 2. A. Biopsia pulmonar transbronquial. Compromiso pulmonar por histiocitosis de Langerhans (PLCH): quistes incipientes (flecha roja) al lado de cicatriz estrellada (flecha negra). B. Biopsia pulmonar transbronquial. Compromiso pulmonar por histiocitosis de Langerhans (PLCH): nódulos acompañados de células inflamatorias. C. Biopsia pulmonar transbronquial. Compromiso pulmonar por histiocitosis de Langerhans (PLCH): eosinófilos (flecha negra) y células de Langerhans (flecha roja). D. Biopsia pulmonar transbronquial. Compromiso pulmonar por histiocitosis de Langerhans (PLCH): inmunohistoquímica positiva para CD1a (células de Langerhans).

Hasta la mitad de los pacientes suele presentar patrones espirométricos normales, seguido de alteraciones restrictivas y obstructivas cuya predominancia varía en las distintas series des$\operatorname{critas}^{8,9}$. La mayoría presenta disminución de la $\mathrm{DLCO}^{14}$.

La hipertensión pulmonar (HTP) es una complicación grave y común en la PLCH avanzada, y estaría relacionada a alteraciones vasculares primarias independientes del compromiso intersticial o de la vía área, por lo que el estudio ecocardiográfico se recomienda como tamizaje $\mathrm{e}^{15,16}$.

En la tomografía computarizada (TC) de tórax, la PLCH se caracteriza por compromiso peribronquiolar de predominio en las zonas superiores y medias del pulmón, por nódulos con bordes irregulares de 1-10 mm que pueden cavitarse. En fases avanzadas predominan las formaciones fibroquísticas y el enfisema paracicatricial. La combinación de múltiples quistes, con predominio de las zonas medias y altas en un fumador joven, pueden ser diagnósticas de $\mathrm{PLCH}^{8-11}$.

Se ha descrito que un lavado bronquioalveolar (LBA) con al menos $5 \%$ de células CD1a+ (receptor identificado como marcador específico para células de Langerhans) en el contexto clínico adecuado podría ser diagnóstico, pese a que la detección de un porcentaje menor no excluye el diagnóstico ${ }^{17}$.

La utilización de biopsias transbronquiales (BTB) y/o quirúrgicas deberían reservarse sólo para aquellos casos en que la tomografía computarizada no presente los hallazgos típicos.

Dentro de los hallazgos histológicos se descri- ben nódulos celulares bronquiolocéntricos compuestos por CL y células inflamatorias (linfocitos, macrófagos, eosinófilos, células plasmáticas y neutrófilos), con destrucción de la pared bronquiolar y el parénquima adyacente, acompañados de grados variables de inflamación, en las fases más avanzadas predominarían las formaciones quísticas y fibrosas, incluyendo enfisema junto a las cicatrices. El estudio inmunohistoquímico con CD1a y CD207 es específico para el diagnóstico ${ }^{8,17}$.

El tratamiento más importante es el cese del tabaquismo, con lo que se logra estabilizar o mejorar el compromiso pulmonar por la enfermedad, pero en algunos casos la enfermedad progresa. No existen marcadores pronósticos para predecir la evolución de la enfermedad.

Los corticoesteroides han sido utilizados en casos de enfermedad grave o progresiva, pero no existe evidencia sistematizada sobre su utilidad. Otros inmunosupresores como ciclofosfamida, metrotrexato y etopósido se han utilizado en aquellos casos sin respuesta a esteroides o enfermedad multiorgánica. La cladribina, un agente citotóxico con acción selectiva en los linfocitos y monocitos, ha sido reportado como útil en algunos casos de enfermedad agresiva multisistémica.

Si la HTP es una complicación clínicamente relevante, debe considerarse su tratamiento farmacológico específico.

El trasplante pulmonar debería ser planteado en pacientes con enfermedad avanzada y compromiso respiratorio severo o HTP severa, pese a que la enfermedad podría recurririr, ${ }^{9}, 18$. 


\section{Bronquiolitis respiratoria}

\section{Caso clínico}

Mujer de 38 años, abogada, procedente de Copiapó, fumadora (IPA 20), antecedente familiar de asma. Historia de frecuentes episodios de infecciones respiratorias altas catalogadas como resfríos. Consulta por cuadro de 1 mes de evolución caracterizado por tos intensa y persistente.

TC tórax (Figuras $3 \mathrm{~A}$ y B).

Pruebas de función pulmonar: Espirometría: CVF $3.990 \mathrm{~mL} \mathrm{VEF}{ }_{1} 2.930 \mathrm{~mL} \mathrm{VEF}_{1} / \mathrm{CVF} 73 \%$ DLCO: $37 \%$ del teórico.

Se realiza LBA con biopsia transbronquial, se alcanzan a tomar solo dos muestras al complicarse por un neumotórax. Posteriormente se practica una videotoracoscopía, donde se obtiene biopsia pulmonar (Figura 4 A y B) que informó; parénquima de arquitectura conservada con leve relleno alveolar peribronquiolar por macrófagos con pigmento, vía aérea deformada con fibrosis y ensanchamiento de su pared como expresión de daño inhalatorio, hallazgos concordantes con bronquiolitis respiratoria.

Se le indicó Prednisona $30 \mathrm{mg}$ diarios que mantiene durante un mes, con lo cual logra remisión clínica e imagenológica.

\section{Comentarios}

La bronquiolitis respiratoria (BR) es una expresión histológica de la respuesta inflamatoria crónica a la inhalación de humo de tabaco (actual o previa). Por sí sola suele ser asintomática, en- contrándose en biopsias quirúrgicas pulmonares (realizadas por otros motivos) de prácticamente todos los fumadores. Si las alteraciones son lo suficientemente marcadas como para reflejarse en un estudio imagenológico (compromiso difuso) y alterar las pruebas funcionales, con o sin síntomas, se denomina "bronquiolitis respiratoria asociada a una enfermedad pulmonar difusa" (BR-EPD). Se han descrito casos aislados de BR-EPD en no fumadores, incluyendo a sujetos expuestos a tabaquismo pasivo ${ }^{7,19-24}$.

Existe leve predominio en hombres. La edad de inicio de los síntomas fluctúa entre los 22 y 70 años, y ocurre más precozmente mientras mayor sea el consumo de tabaco. Generalmente se asocia a un índice paquete año (IPA) mayor a $30^{20,21,27}$.

La mayoría se presenta en forma insidiosa (semanas a meses) con disnea de esfuerzos y tos, que suelen ser leves. Esta clínica puede enmascararse si se asocia a bronquitis crónica asociada a tabaco. Al examen físico, las crepitaciones bibasales al final de la inspiración son el hallazgo más común ${ }^{7,21,24}$.

En cuanto a las PFP, prima un patrón restrictivo, pero también puede existir obstrucción leve, con una capacidad de difusión ligeramente reducida ${ }^{7}$.

Los hallazgos radiográficos usualmente son sutiles, por lo que la TC tórax de alta resolución es lo más utilizado (Figuras 3 A y B). Típicamente se observan opacidades nodulares centrolobulillares y vidrio esmerilado en parches, con
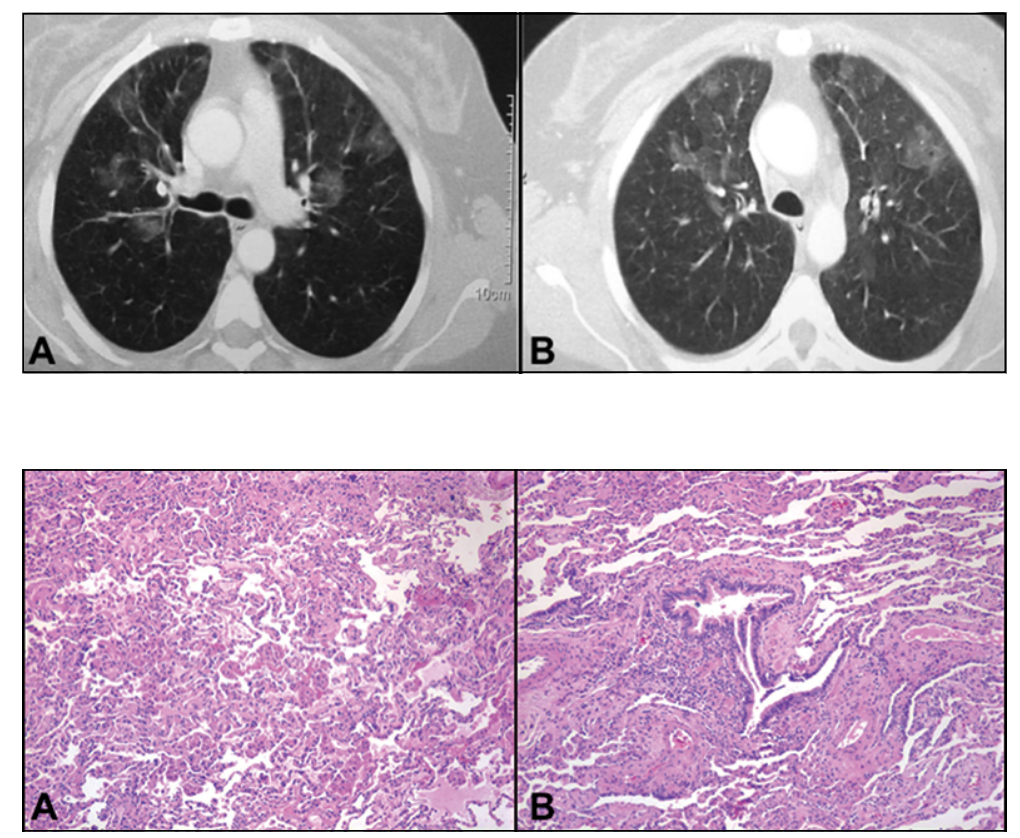

Figura 3 A y B. TC Tórax sin contraste. Bronquiolitis respiratoria $(\mathrm{BR})$ : enfermedad pulmonar difusa centroacinar, con predominio en lóbulos superiores. Arquitectura conservada y tenue aumento de densidad parcialmente confluente.

Figura 4. A. Biopsia pulmonar por VTC. Bronquiolitis Respiratoria (BR): pulmón de arquitectura conservada con leve relleno alveolar peribronquiolar por macrófagos con pigmento. B. Biopsia pulmonar por VTC. Bronquiolitis Respiratoria (BR): vía aérea deformada con fibrosis y ensanchamiento de su pared como expresión de daño inhalatorio. 
engrosamiento de la pared bronquial. Además, se ha descrito enfisema en lóbulos superiores, y áreas en mosaico y leve reticulado en los lóbulos inferiores principalmente. Radiológicamente, el principal diagnóstico diferencial es la neumonitis por hipersensibilidad subaguda, que raras veces afecta a fumadores (actuales o previos). El LBA cumple un rol en esta disyuntiva, pues la observación de los llamados "macrófagos del fumador" (contienen pigmentos marrones derivados del tabaco) y la ausencia de linfocitosis sugiere el diagnóstico de BR-EPD, y descarta una neumonitis por hipersensibilidad $7,25,26$.

La biopsia pulmonar es pocas veces necesaria. El hallazgo más característico es el conglomerado bronquiolocéntrico de "macrófagos del fumador", extendiéndose hacia los espacios alveolares adyacentes. Además, se asocia a engrosamiento peribronquiolar variable, y la fibrosis intersticial es mínima o ausente. Cabe destacar que la BREPD y el DIP representan un mismo espectro histológico (de acumulación de macrófagos). Sin embargo, se distinguen por la extensión/distribución de tal acumulación, patrón tomográfico, presentación clínica y respuesta a la terapia. Por tales razones se clasifican como entidades separadas $7,25,27$.

Respecto al manejo, suspender el hábito tabáquico tiene un rol central. El tiempo de recupera- ción después del cese del tabaquismo varía entre 1 a 30 años. En casos sintomáticos, si la enfermedad no remite en forma significativa, puede intentarse un curso de esteroides después de analizar minuciosamente los riesgos y beneficios ${ }^{3,21,22,24,28}$.

El pronóstico generalmente es favorable y la sobrevida a largo plazo es la regla. Sin embargo, en la literatura hay casos descritos con evolución más tórpida ${ }^{20,21}$.

\section{Neumonia intersticial descamativa}

\section{Caso clínico}

Hombre de 56 años, proveniente de Copiapó, transportista de materiales de construcción, fumador activo (IPA 45). Cuadro clínico caracterizado por tos crónica y disnea de esfuerzo de magnitud moderada de años de evolución, con exacerbación en las últimas semanas previas a la consulta.

Al examen físico destacan escasos crépitos basales bilaterales e hipocratismo digital.

TC tórax (Figura 5 A y B).

Pruebas de función pulmonar: Espirometría: CVF $4.380 \mathrm{~mL} \mathrm{VEF}_{1} 2.810 \mathrm{~mL} \mathrm{VEF}_{1} / \mathrm{CVF}$ 64\% DLCO: $30 \%$ del teórico.

Se realiza biopsia pulmonar por videotoracoscopía (VTC), extrayéndose muestra de los lóbulos superior e inferior derecho. Biopsia: Figura 6 A, B y C.

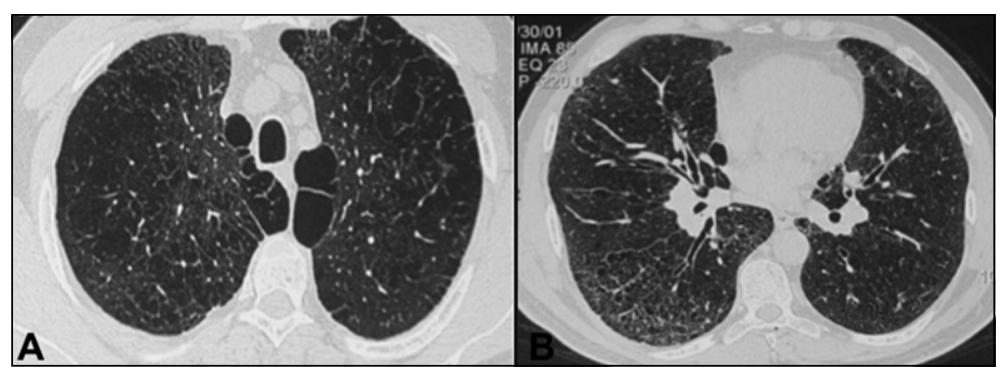

Figura 5 A y B. TC Tórax sin contraste. Neumonia Intersticial Descamativa (DIP): enfisema centroacinar, con bulas marginales superiores, aumento difuso de la densidad radiológica de predominio basal central y cortical.

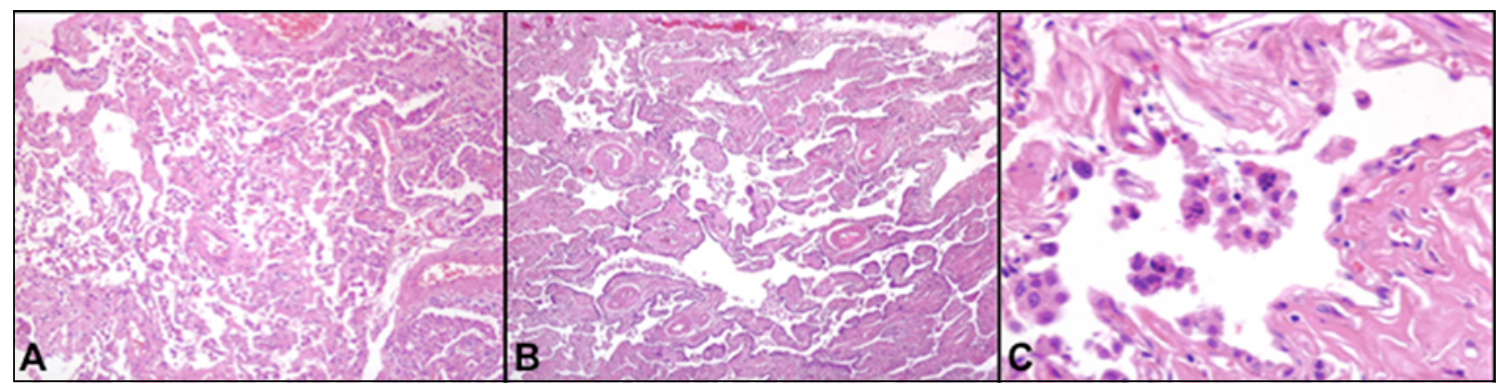

Figura 6. A y B. Biopsia pulmonar por VTC. Neumonia Intersticial Descamativa (DIP): pulmón de arquitectura conservada con extensas imágenes de relleno alveolar caracterizadas por la presencia de macrófagos, áreas de ensanchameinto de las paredes alveolares con extensa metaplasia bronquiolar que traducen daño inhalatorio de la vía aérea y el parénquima pulmonar vecino. C. Biopsia pulmonar por VTC. Neumonia Intersticial Descamativa (DIP): macrófagos con pigmento acompañados de escasos eosinófilos en un espacio aéreo. 
Posteriormente se inició tratamiento con prednisona $40 \mathrm{mg} /$ día, que se mantuvo durante un mes y se redujo lentamente hasta $10 \mathrm{mg} /$ día. Además, dejó de fumar. Aproximadamente 3-4 meses post inicio de corticoterapia notó mejoría sintomática. En este contexto, se repiten las pruebas de función pulmonar: CVF $4.090 \mathrm{~mL} \mathrm{VEF}_{1} 2.580$ $\mathrm{mL} \mathrm{VEF}_{1} / \mathrm{CVF}$ 63\%. DLCO: 45\% del teórico. En vista de la mejoría clínica y de la DLCO, se decide mantener con prednisona $10 \mathrm{mg}$ diarios por tiempo indefinido.

\section{Comentarios}

La neumonía intersticial descamativa (DIP) representa el espectro final de la BR-EPD, con hallazgos histológicos similares y asociación casi invariable al tabaquismo (>90\%). Los casos de DIP asociados a otras condiciones (enfermedades autoinmunes, infecciones o ingesta de drogas) son muy inusuales. Es más frecuente en hombres, en la cuarta a sexta década, y los principales síntomas de presentación son la disnea y tos seca, de inicio insidioso. Al examen físico, destacan las crepitaciones $(60 \%)$ y la acropaquia $(50 \%)^{29-32}$.

Respecto a las PFP, la alteración de la DLCO es la más común y llamativa. No es raro que las reducciones de DLCO sean mayores al 50\%, orientando a la severidad de la enfermedad subyacente. Alteraciones restrictivas en la espirometría, también son comunes, mientras que la hipoxemia sólo aparece en estados avanzados de la enfermedad ${ }^{7,32}$.

El hallazgo más frecuente en la TC (Figura 5) es la presencia de vidrio esmerilado, generalmente bilateral, relativamente simétrico, "parcheado" o difuso, de predominio subpleural y bibasal ( $2 / 3$ basales). En los casos avanzados, pueden encontrarse bronquiectasias por tracción o panal de abejas (usualmente ausente) ${ }^{7,32-35}$.

En la histología, es característico el relleno de los espacios alveolares por "macrófagos del fumador", con compromiso pulmonar difuso y uniforme. La arquitectura pulmonar generalmente está preservada, y puede existir un tenue infiltrado inflamatorio crónico en el intersticio ${ }^{7}$.

Respecto al tratamiento, la primera medida es suspender el tabaco. La administración de corticoides sistémicos generalmente logra la estabilización, rara vez una mejoría. En los casos que no exista respuesta a esteroides, puede considerarse la adición de ciclofosfamida o azatioprina. Sin embargo, cabe destacar que la evidencia del efecto de las drogas es escasa, y basada esencialmente en estudios retrospectivos, observacionales y reporte de $\operatorname{casos}^{31}$.

Dos tercios de los pacientes que no reciben tratamiento presentarán progresión de la enfermedad. De los pacientes tratados, hasta un cuarto de ellos continúa deteriorándose a pesar de la terapia, pudiendo incluso llegar a requerir trasplante pulmonar ${ }^{7,31}$.

La progresión a fibrosis pulmonar severa es poco común, aunque la coexistencia con patrón de neumonía intersticial no específica es frecuente. La sobrevida a 10 años es aproximadamente un $70 \%$ (rango de mortalidad fluctúa entre un 6 y $28 \%)^{7,31,36}$.

\section{Fibrosis intersticial relacionada a tabaco}

\section{Caso clínico}

Mujer de 49 años, fumadora activa (IPA 35). Dentro del estudio de un cuadro respiratorio, se realiza radiografía de tórax que evidencia EPD, por lo que se deriva a nuestro centro para estudio. $\mathrm{Al}$ examen físico destacan crépitos hacia las bases y acropaquia.

TC tórax (Figuras 7 A y B).

Pruebas de función pulmonar: Espirometría: CVF $2.440 \mathrm{~mL}$ (82\%); DLCO: 51\% del teórico, C6M: 558 m (94\%) Sat 98\% - 95\%. Estudio reumatológico negativo.

Por sospecha de DIP, se inicia tratamiento con Prednisona $0,5 \mathrm{mg} / \mathrm{kg} /$ día y se intenta cese de tabaquismo. A los 3 meses, desarrolló hipertensión arterial y diabetes mellitus, por lo que se suspenden los esteroides. Control con TC Tórax a los 18 meses evidencia progresión de la EPD, por lo que se reinician corticoides con nula respuesta, que se suspenden posteriormente.

A los tres años de control se solicitan nuevas PFP: CVF $2.920 \mathrm{~mL}$ (76\%), DLCO: $42 \%$ del teórico.

Por EPD de etiología no precisada se solicita biopsia pulmonar por VTC (videotoracoscopia) que informa engrosamiento de la pared bronquiolar con deformación de la misma y presencia de macrófagos con pigmento de tabaco en el lumen bronquiolar y los espacios alveolares periféricos, algunas zonas de parénquima subpleural con fibrosis, compatible con fibrosis intersticial relacionada a tabaco (SRIF) (Figura 8).

Con el diagnóstico confirmado de SRIF, se decide observar y continuar en controles ambulatorios periódicos.

\section{Comentarios}

La SRIF, es una entidad recientemente descrita, común en fumadores. El término SRIF fue inicialmente utilizado para describir cambios parenquimatosos en muestras de lobectomía de 


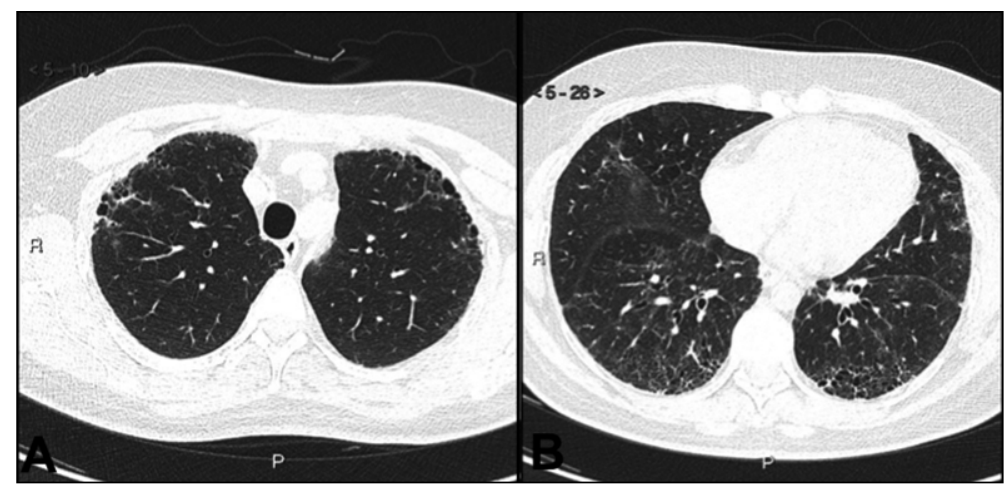

Figura 7 A y B. TC Tórax sin contraste. Fibrosis intersticial relacionada a tabaco (SRIF): imágenes quísticas corticales más discretamente superiores, que corresponden a bulas marginales, reforzadas por parénquima que presenta reacción probablemente fibrosa, formando quistes de pared definida. Hacia las bases el compromiso es más significativo extendiéndose por ramificaciones broncovasculares hacia el interior. Adicionalmente se asocian tenues focos con aumento de la densidad radiológica en regiones centrales de aspecto inflamatorio, que pueden corresponder a fenómenos de bronquiolitis respiratoria asociada.

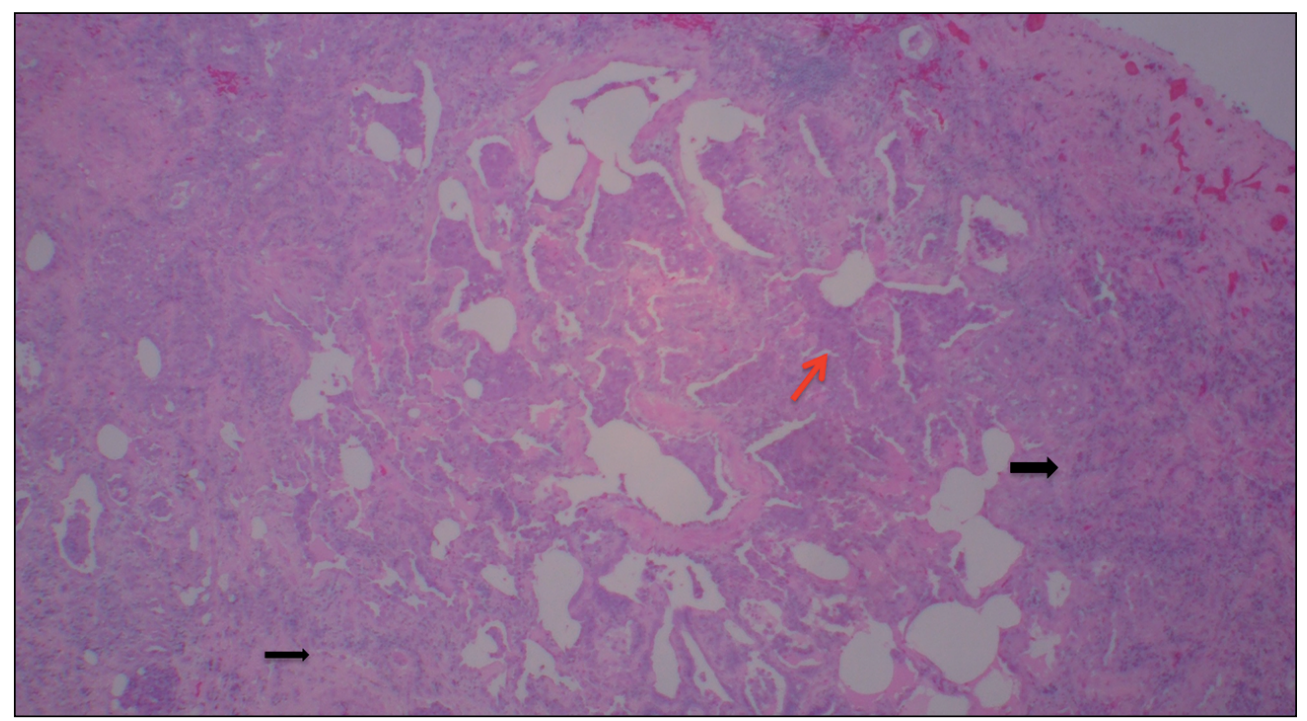

Figura 8. Biopsia pulmonar por VTC. Fibrosis intersticial relacionada a tabaco (SRIF): pulmón con fibrosis terminal (flecha negra) asociado a presencia de macrófagos con pigmento en espacios alveolares (flecha roja).

pacientes con cáncer, con áreas de importante fibrosis acompañada por bronquiolitis y enfisema, pero que no tendría una traducción clínica en la mayoría de los pacientes ${ }^{37}$.

La edad al momento del diagnóstico es variable y va de los 32 a los 81 años (promedio 51 años en la serie de Katzenstein y 38 años en la serie de Yousem), todos ellos fumadores activos o suspendidos, sin diferencias estadísticas por sexo, con un IPA promedio de $38^{38,39}$.

El estudio funcional en esta condición puede ser obstructivo con leve disminución de la $\mathrm{DLCO}^{40,41}$.

La TC de tórax evidencia infiltrados microno- dulares y opacidades en vidrio esmerilado con distribución en parches, en general con compromiso mayor en las zonas más altas del pulmón ${ }^{41}$.

Los hallazgos histológicos característicos corresponden a fibrosis con engrosamiento de los septos alveolares por depósito de colágeno con mínima inflamación asociada, especialmente de localización subpleural donde se asocia a enfisema, pero puede afectar a porciones más profundas. Es constante la presencia de macrófagos pigmentados intra-alveolares, indicadores de bronquiolitis respiratoria, generalmente numerosos. Algunos autores han descrito la presencia de focos fibroblásticos ${ }^{38,42}$. 
No se han descrito estrategias terapéuticas específicas para esta entidad, pero como en todas las EPD asociadas a tabaco, la suspensión del tabaquismo debiese plantearse desde el momento mismo de la sospecha.

Respecto al pronóstico, todos los pacientes descritos en las series publicadas estaban vivos al terminar el tiempo de seguimiento ( 2 meses a 3,2 años), la mayoría de ellos con una enfermedad estable ${ }^{38,39,41}$.

\section{Otras EPD asociadas a tabaquismo}

\section{Neumonía intersticial usual (UIP)}

Más frecuente en hombres, mayores de 50 años. La disnea de esfuerzo y tos seca son los principales síntomas de presentación. Al examen físico destacan los crépitos $(70 \%)$ y acropaquia (30-50\%). En la espirometría, trastorno restrictivo, con DLCO disminuida. Típicamente el hallazgo en la TC es un patrón tipo UIP con infiltrados reticulares de predominio basal y periférico, panal de abejas y/o bronquiectasias por tracción en ausencia de hallazgos inconsistentes con UIP. En el LBA puede existir leve neutrofilia y eosinofilia; y en la histología, fibrosis "en parches" y/o destrucción de la arquitectura, con o sin panal de abeja, de distribución predominantemente subpleural y/o paraseptal. Los focos de fibroblastos también son característicos. El tratamiento con antifibróticos, podría estabilizar o disminuir la progresión de la enfermedad ${ }^{43,44}$.

\section{Neumonía eosinofilica aguda (AEP)}

Puede desarrollarse tanto en nuevos fumadores como en aquellos que vuelven a fumar después de un período de abstinencia o que aumentaron su consumo. También se ha descrito con el uso combinado de tabaco y marihuana o cannabis. La respuesta al tratamiento con corticoesteroides sistémicos suele ser excelente, pudiendo obtenerse remisión completa ${ }^{45-47}$.

\section{Artritis reumatoide asociada a EPD (AR-EPD)}

$\mathrm{Su}$ momento de inicio y curso son independientes de la enfermedad subyacente. Las manifestaciones son heterogéneas, y el plan de tratamiento debe elaborarse de forma individual, en estrecha cooperación con reumatología ${ }^{48-50}$.

\section{Hemorragia alveolar difusa en síndrome de Goodpasture (HAD en SG)}

Una serie de 51 pacientes con síndrome de Goodpasture (SG) mostró una clara asociación
$(100 \%)$ entre el "estado del tabaquismo" y la probabilidad de ocurrencia de HAD. Por lo tanto, la inhalación de humo de tabaco debe considerarse como un factor de riesgo para la aparición de $\mathrm{HAD}$ en SG. El tratamiento en el cuadro agudo requiere un equipo multidisciplinario, e incluye los inmunosupresores, plasmaféresis, $y$, en presencia de insuficiencia renal, hemodiálisis ${ }^{51-53}$.

\section{Proteinosis alveolar (PA)}

Un $56-79 \%$ de los pacientes con PA tienen antecedente de tabaquismo actual o previo. En casos de deterioro significativo de la función pulmonar, el tratamiento de elección es el lavado pulmonar total. Si no hay respuesta satisfactoria y se demuestra la presencia de anticuerpos anti factor estimulante de colonias de granulocitos y macrófagos (GM-CSF), puede evaluarse la terapia de inhalación con GM-CSF ${ }^{54-58}$.

\section{Discusión}

Aunque las EPD son relativamente poco comunes, representan una carga importante para la salud (tanto a nivel personal como colectivo), ya que frecuentemente afecta a adultos jóvenes (edades de mayor productividad). Con el aumento creciente del consumo de tabaco, sobre todo en países en desarrollo, es probable que esta carga descrita siga en alza.

Debe considerarse la presencia de EPD-TBQ en pacientes con historia de tabaquismo y síntomas respiratorios inespecíficos como disnea o tos seca. Se ha sugerido que el fumar también puede provocar anormalidades pulmonares intersticiales inespecíficas. Varios estudios de cribado para la identificación precoz de cáncer de pulmón mediante TC de baja dosis han demostrado que casi el 10\% de los fumadores puede presentar alteraciones pulmonares intersticiales, que pueden progresar si continúa fumando ${ }^{59-61}$. En general, estudio con TC de tórax de alta resolución y la histología, son los elementos principales para diferenciar entre las distintas EPD-TBQ descritas hasta el momento.

Si bien la mayoría de estas enfermedades son de pronóstico benigno, muchas veces con estabilización de la enfermedad luego de la suspensión del hábito tabáquico, otras pueden progresar hasta la insuficiencia respiratoria crónica. Dada la escasa literatura existente sobre el tratamiento farmacológico, la suspensión del hábito tabáquico es la gran medida terapéutica sugerida. 


\section{Bibliografía}

1.- TRAVIS W, COSTABEL U, HANSELL D, KING T, LYNCH D, NICHOLSON A, et al. An official American Thoracic Society/European Respiratory Society statement: Update of the international multidisciplinary classification of the idiopathic interstitial pneumonias. Am J Respir Crit Care Med 2013; 188: 733-48.

2.- BAUMGARTNER K, SAMET J, STIDLEY C, COLBY T, WALDRON J. Cigarette smoking: a risk factor for idiopathic pulmonary fibrosis. Am J Respir Crit Care Med 1997; 155: 242-8.

3.- WELLS A, NICHOLSON A, HANSELL D. Challenges in pulmonary fibrosis: smoking-induced diffuse interstitial lung diseases. Thorax 2007; 62: 904-10.

4.- FRANKS T, GALVIN J. Smoking-Related "Interstitial" Lung Disease. Arch Pathol Lab Med 2015; 139: 974-7

5.- MORLÁ M, BUSQUETS X, PONS J, SAULEDA J, MACNEE W, AGUSTÍ A. Telomere shortening in smokers with and without COPD. Eur Respir J 2006; 27: $525-8$

6.- PATEL A, LIN L, GEYER A, HASPEL J, AN C, CAO J, et al. Autophagy in idiopathic pulmonary fibrosis. PLoS ONE 2012; 7: e41394.

7.- MARGARITOPOULOS G, HARARI S, CAMINATI A, ANTONIOU K. Smoking-related idiopathic interstitial pneumonia: A review. Respirology 2016; 21: 57-64.

8.- RODEN A, YI E. Pulmonary Langerhans Cell Histiocytosis: An update from the Pathologists' Perspective. Arch Pathol Lab Med 2016; 140: 230-40.

9.- ELIA D, TORRE O, CASSANDRO R, CAMINATI A, HARARI S. Pulmonary Langerhans cell histiocytosis: a comprehensive analysis of 40 patients and literature review. Eur J Int Med 2015; 26: 351-6.

10.- GAENSLER E, CARRINGTON C. Open biopsy for chronic diffuse infiltrative lung disease: clinical, roentgenographic, and physiological correlations in 502 patients. Ann Thorac Surg 1980, 30: 411-26.

11.- VASSALLO R, RYU J, COLBY T, HARTMAN T, LIMPER AH. Pulmonary Langerhans'-cell histiocytosis. N Engl J Med 2000; 342: 1969-78.

12.- MASON R, FOLEY N, BRANLEY H, ADAMALI H, HETZEL M, MAHER T, et al. Pulmonary Langerhans cell histiocytosis (PLCH): a new UK register. Thorax 2014; 69: 766-7.

13.- SURI H, YI E, NOWAKOWSKI G, VASSALLO R. Pulmonary langerhans cell histiocytosis. Orphanet J Rare Dis 2012; 7: 16.

14.- VASSALLO R, RYU J, SCHROEDER D, DECKER P, LIMPER A. Clinical outcomes of pulmonary Langerhans' cell histiocytosis in adults. N Engl J Med 2002; 346: 484-90.

15.- DAURIAT G, MAL H, THABUT G, MORNEX J, BERTOCCHI M, TRONC F, et al. Lung transplantation for pulmonary Langerhans' cell histiocytosis: a multicenter analysis. Transplantation 2006; 81: 746-50.
16.- FARTOUKH M, HUMBERT M, CAPRON F, MAÎTRE S, PARENT F, LE GALL C, et al. Severe pulmonary hypertension in histiocytosis X. Am J Respir Crit Care Med 2000; 161: 216-23.

17.- BAQIR M, VASSALLO R, MALDONADO F, YI E, RYU J. Utility of bronchoscopy in pulmonary. Langerhans cell histiocytosis. J Bronchol Interv Pulmonol 2013; 20: 309-12.

18.- LORILLON G, BERGERON A, DETOURMIGNIES L, JOUNEAU S, WALLAERT B, FRIJA J, et al. Cladribine is effective against cystic pulmonary Langerhans cell histiocytosis. Am J Respir Crit Care Med 2012; 186: 930-2.

19.- CAMINATI A, GRAZIANO P, SVERZELLATI N, HARARI S. Smoking-related interstitial lung diseases. Pathologica 2010; 102: 525-36.

20.- RYU J, MYERS J, CAPIZZI S, DOUGLAS W, VASSALLO R, DECKER P. Desquamative interstitial pneumonia and respiratory bronchiolitis-associated interstitial lung disease. Chest 2005; 127: 178-84.

21.- PORTNOY J, VERALDI K, SCHWARZ M, COOL C, CURRAN-EVERETT D, CHERNIACK R, et al. Respiratory bronchiolitis-interstitial lung disease: long-term outcome. Chest 2007; 131: 664-71.

22.- HAGMEYER L, RANDERATH W. Smoking-related interstitial lung disease. Dtsch Arztebl Int 2015; 112: 43-50.

23.- WOO O, YONG H, OH Y, LEE S, KIM H, KANG E. Respiratory bronchiolitis-associated interstitial lung disease in a nonsmoker: radiologic and pathologic findings. AJR 2007; 188: 412-4.

24.- SIEMINSKA A, KUZIEMSKI K. Respiratory bronchiolitis-interstitial lung disease. Orphanet J Rare Dis 2014; 9:106.

25.- ATTILI A, KAZEROONI E, GROSS B, FLAHERTY K, MYERS J, MARTÍNEZ F. Smoking-related interstitial lung disease: radiologic-clinical-pathologic correlation. Radiographics 2008; 28: 1383-96.

26.- HANSELL D, NICHOLSON A. Smoking-related diffuse parenchymal lung disease: HRCT-pathologic correlation. Semin Respir Crit Care Med 2003; 24: 377-92.

27.- CAMINATI A, GRAZIANO P, SVERZELLATI N, HARARI S. Smoking-related interstitial lung diseases. Pathologica 2010; 102: 525-36.

28.- PARK J, BROWN K, TUDER R, HALE V, KING T, LYNCH D. Respiratory bronchiolitis-associated interstitial lung disease: radiologic features with clinical and pathologic correlation. J Comput Assist Tomogr 2002; 26: $13-20$.

29.- TAZELAAR H, WRIGHT J, CHURG A. Desquamative interstitial pneumonia. Histopathology 2011; 58: 50916.

30.- CAMINATI A, CAVAZZA A, SVERZELLATI N, HARARI S. An integrated approach in diagnosis of smoking-related interstitial lung diseases. Eur Respir Rev 2012; 21: 207-17. 
31.- CARRINGTON C B, GAENSLER E A, COUTU R E, FITZGERALD M X, GUPTA R G. Natural history and treated course of usual and desquamative interstitial pneumonia. N Engl J Med 1978; 298: 801-9.

32.- RYU J H, COLBY T V, HARTMAN T E, VASSALLO R. Smoking-related interstitial lung diseases: a concise review. Eur Respir J 2001; 17: 122-132.

33.- HARTMAN T E, PRIMACK S L, SWENSEN S J, HANSELL D, MCGUINNESS G, MULLER N L. Desquamative interstitial pneumonia: thin-section CT findings in 22 patients. Radiology 1993; 187: 787-90.

34.- AKIRA M, YAMAMOTO S, HARA H, SAKATANI M, UEDA E. Serial computed tomographic evaluation in desquamative interstitial pneumonia. Thorax 1997; 52: 333-7.

35.- DESAI S R, RYAN S M, COLBY T V. Smoking-related interstitial lung diseases: histopathological and imaging perspectives. Clin Radiol 2003; 58: 259-68.

36.- YOUSEM S A, COLBY T V, GAENSLER E A. Respiratory bronchiolitis-associated interstitial lung disease and its relationship to desquamative interstitial pneumonia. Mayo Clin Proc 1989; 64: 1373-80.

37.- KATZENSTEIN A-LA, MUKHOPADHYAY S, ZANARDI C, DEXTER E. Clinically occult interstitial fibrosis in smokers: classification and significance of a surprisingly common finding in lobectomy specimens. Hum Pathol 2010; 41: 316-25.

38.- KATZENSTEIN A-LA. Smoking-related interstitial fibrosis (SRIF): pathologic findings and distinction from other chronic fibrosing lung diseases. J Clin Pathol 2013; 66: 882-7.

39.- YOUSEM S A. Respiratory bronchiolitis-associated interstitial lung disease with fibrosis is a lesion distinct from fibrotic nonspecific interstitial pneumonia: a proposal. Mod Pathol 2006; 19: 1474-1479.

40.- EL-KERSH K, PÉREZ R L, SMITH J S, FRAIG M. Smoking-related interstitial fibrosis (SRIF) and pulmonary hypertension. BMJ Case Rep 2013; 2013: doi: 10.1136/bcr-2013-008970.

41.- REDDY T L, MAYO J, CHURG A. Respiratory bronchiolitis with fibrosis: high-resolution computed tomography findings and correlation with pathology. Ann Am Thorac Soc 2013; 10: 590-601

42.- KATZENSTEIN A L. Smoking-related interstitial fibrosis (SRIF), pathogenesis and treatment of usual interstitial pneumonia (UIP), and transbronchial biopsy in UIP. Mod Pathol 2012; 25(Suppl 1): S68-S78.

43.- BEHR J, GÜNTHER A, AMMENWERTH W, BITTMANN I, BONNET R, BUHL R, et al. German guideline for diagnosis and management of idiopathic pulmonary fibrosis. Pneumologie 2013; 67: 81-111.

44.- RAGHU G, COLLARD H R, EGAN J J, MARTÍNEZ F J, BEHR J, BROWN K K, et al. An official ATS/ ERS/JRS/ALAT statement: idiopathic pulmonary fibrosis: evidence-based guidelines for diagnosis and management. Am J Respir Crit Care Med 2011; 183 : 788-824.

45.- UCHIYAMA H, SUDA T, NAKAMURA Y, SHIRAI M, GEMMA H, SHIRAI T, et al. Alterations in smoking habits are associated with acute eosinophilic pneumonia. Chest 2008; 133: 1174-80.

46.- RHEE C K, MIN K H, YIM N Y, LEE J E, LEE N R, CHUNG M P, et al. Clinical characteristics and corticosteroid treatment of acute eosinophilic pneumonia. Eur Respir J 2013; 41: 402-9.

47.- SHORR A F, SCOVILLE S L, CERSOVSKY S B, SHANKS G D, OCKENHOUSE C F, SMOAK B L, et al. Acute eosinophilic pneumonia among US Military personnel deployed in or near Iraq. JAMA 2004; 292 : 2997-3005.

48.- MIKULS T R, SAYLES H, YU F, LEVAN T, GOULD $\mathrm{K}$ A, THIELE G M, et al. Associations of cigarette smoking with rheumatoid arthritis in African Americans. Arthritis Rheum 2010; 62: 3560-8.

49.- KELLY C A, SARAVANAN V, NISAR M, ARTHANARI S, WOODHEAD F A, PRICE-FORBES A N, et al. Rheumatoid arthritis-related interstitial lung disease: associations, prognostic factors and physiological and radiological characteristics-a large multicentre UK study. Rheumatology (Oxford) 2014; 53: 1676-82.

50.- HUTCHINSON D, SHEPSTONE L, MOOTS R, LEAR J T, LYNCH M P. Heavy cigarette smoking is strongly associated with rheumatoid arthritis (RA), particularly in patients without a family history of RA. Ann Rheum Dis 2001; 60: 223-7.

51.- DONAGHY M, REES A J. Cigarette smoking and lung haemorrhage in glomerulonephritis caused by autoantibodies to glomerular basement membrane. Lancet 1983; 2: $1390-3$.

52.- CHAN A L, LOUIE S, LESLIE K O, JUAREZ M M, ALBERTSON T E. Cutting edge issues in Goodpasture's disease. Clin Rev Allergy Immunol 2011; 41: 151-62.

53.- LEATHERMAN J W, DAVIES S F, HOIDAL J R. Alveolar hemorrhage syndromes: diffuse microvascular lung hemorrhage in immune and idiopathic disorders. Medicine (Baltimore) 1984; 63: 343-61.

54.- INOUE Y, TRAPNELL B C, TAZAWA R, ARAI T, TAKADA T, HIZAWA N, et al. Characteristics of a large cohort of patients with autoimmune pulmonary alveolar proteinosis in Japan. Am J Respir Crit Care Med 2008; 177: 752-62.

55.- TAZAWA R, TRAPNELL B C, INOUE Y, ARAI T, TAKADA T, NASUHARA Y, et al. Inhaled granulocyte/ macrophage colony stimulating factor as therapy for pulmonary alveolar proteinosis. Am J Respir Crit Care Med 2010; 181: 1345-54.

56.- BONELLA F, BAUER P C, GRIESE M, OHSHIMO S, GUZMÁN J, COSTABEL U. Pulmonary alveolar proteinosis: new insights from a single-center cohort of 70 patients. Respir Med 2011; 105: 1908-16. 
57.- SEYMOUR J F, PRESNEILL J J. Pulmonary alveolar proteinosis: progress in the first 44 years. Am J Respir Crit Care Med 2002; 166: 215-35.

58.- HUIZAR I, KAVURU M S. Alveolar proteinosis syndrome: pathogenesis, diagnosis, and management. Curr Opin Pulm Med 2009; 15: 491-8.

59.- SVERZELLATI N, GUERCI L, GIORGIA R, CALABRÒ E, LA VECCHIA C, MARCHIANÒ A, et al. Interstitial lung diseases in a lung cancer screening trial. Eur Respir J 2011; 38: 392-400.
60.- WASHKO G R, HUNNINGHAKE G M, FERNÁNDEZ IE, NISHINO M, OKAJIMA Y, YAMASHIRO T, et al. Lung volumes and emphysema in smokers with interstitial lung abnormalities. N Engl J Med 2011; 364 : 897-906.

61.- JIN G Y, LYNCH D, CHAWLA A, GARG K, TAMMEMAGI M C, SAHIN H, et al. Interstitial lung abnormalities in a CT lung cancer screening population: prevalence and progression rate. Radiology 2013; 268: 563-71.

Correspondencia a:

Dr. Juan Carlos Rodríguez D.

Instituto Nacional del Tórax

Av. José M. Infante 717. Providencia.

Santiago de Chile.

Email: jcerodriguez@gmail.com 